\title{
RANK-TWO VECTOR BUNDLES ON GENERAL QUARTIC HYPERSURFACES IN $\mathbb{P}^{4}$
}

\author{
Carlo MADONNA
}

\begin{abstract}
In this paper all non-splitting rank-two vector bundles $\mathcal{E}$ without intermediate cohomology on a general quartic hypersurface $X$ in $\mathbb{P}^{4}$ are classified. In particular, the existence of some curves on a general quartic hypersurface is proved.
\end{abstract}

\section{Introduction}

By Horrocks' criterion ([19] pg.39 and [13], [1] and [9]) a rank-two vector bundle on a projective space has no intermediate cohomology (i.e $\left.h^{1}(\mathcal{E}(n))=0 \forall n \in \mathbb{Z}\right)$ if and only if it decomposes as a direct sum of two line bundles. Horrocks' criterion was generalized for the splitting of rank-two vector bundles on quadrics and Grassmannians by Ottaviani ([21]) and for rank-two vector bundles on smooth hypersurfaces in $\mathbb{P}^{4}$ by the author ([17]). In particular in [17] we gave a condition on the number $2 b-c_{1}$ (see Section 1) for a non-splitting rank-two vector bundle without intermediate cohomology on a non singular hypersurface $X$ in $\mathbb{P}^{4}$. Besides the result of Horrocks for projective space, there is such a characterization for smooth quadrics by Knörrer ([16]). In fact, it was shown by Buchweitz, Greuel and Schreyer that on a smooth hypersurface of degree $r$ there is a finite number of non-splitting rank-k vector bundles without intermediate cohomology if and only if $r=1$ or $r=2$ (see [6]). For other threefolds there is a result by Arrondo and Graña ([3]) for the Grassmannian $G(1,4)$ of lines in $\mathbb{P}^{4}$ and a recent result by Arrondo and Costa ([4]) giving a complete classification of all such bundles on some Fano's; in particular they find such a classification for non singular cubic hypersurfaces in $\mathbb{P}^{4}$. There is also a paper by Szurek and

1991 Mathematics Subject Classification: 14F05.

Servicio de Publicaciones. Universidad Complutense. Madrid, 2000 
Wiśniewski ([25]) on moduli of bundles on Fano's coming from conics. More recently we have a result by Costa ([8]) on moduli of vector bundles on ruled Fano threefolds.

It turns out that the first case not known in the literature concerns general quartic hypersurfaces in $\mathbb{P}^{4}$, which are the subject of our analysis. Using the cohomological criteria of [17], the classification of [21] and [4] and a recent result of Iliev and Markushevich ([14]) we are able to classify all non-splitting rank-two vector bundles on such a 3 -fold with no intermediate cohomology.

The main results of our paper is the following:

Main Theorem. Let $\mathcal{E}$ be a non-splitting rank-two vector bundle without intermediate cohomology on a general quartic hypersurface $X \subset \mathbb{P}^{4}$. Then $\mathcal{E}$ is a twist of one of the bundles in the list below:

1. $c_{1}(\mathcal{E})=-1, c_{2}(\mathcal{E})=1$ and $\mathcal{E}$ is associated to a line;

2. $c_{1}(\mathcal{E})=0, c_{2}(\mathcal{E})=2$ and $\mathcal{E}$ is associated to a plane conic;

3. $c_{1}(\mathcal{E})=1, c_{2}(\mathcal{E}) \in\{3,4,5\}$ and $\mathcal{E}$ is associated to a (possibly reducible and non reduced but subcanonical locally Cohen-Macaulay) curve of arithmetic genus 1 and trivial dualizing sheaf;

4. $c_{1}(\mathcal{E})=2, c_{2}(\mathcal{E})=8$ and $\mathcal{E}$ is associated to a "canonical" curve;

5. $c_{1}(\mathcal{E})=3, c_{2}(\mathcal{E})=14$ and $\mathcal{E}$ is associated to a smooth 2-subcanonical curve.

In particular all cases occur on a general quartic hypersurface in $\mathbb{P}^{4}$ (see Section 2).

In Section 2. we will be more precise about the curves that are associated to rank-two bundles of type 4. and 5. above (see Proposition 2.1 and Remark 3).

Some of the examples for proving the existence of these types of bundles on a general quartic hypersurfaces were obtained with an essential use of some computer algebra packages ([7] and [20]).

Our paper is organized in three sections. In Section 1. we recall some generalities and terminology. Section 2. contains examples of non-splitting rank-two vector bundles without intermediate cohomology on non singular quartic hypersurfaces in $\mathbb{P}^{4}$ and a proof of the main 
theorem. We will construct such bundles using the Hartshorne-Serre correspondence between rank-two vector bundles and locally complete intersection subcanonical curves on $X$ (see [10], [12], or [24] for example). Also in the examples we recall some well known and recent results about curves on such a threefold (see [23] and [26] for lines and conics and [14] for half-canonical curves of degree 14 and genus 15), providing a source of curves on $X$. In Section 3. we show the existence of "canonical" and elliptic quintic curves on $X$. In both cases we will use some standard Hilbert scheme techniques as in [15]. Finally we characterize bundles associated to smooth canonical curves and to trigonal canonical curves.

I'd like to thank Prof. L. Chiantini for many useful suggestions, Prof. A. Conca and Prof. F. Orecchia for introducing me to the computational computer packages [7] and [20].

\section{Generalities}

We work over the complex field $\mathbb{C}$. If $Y \subset X$ is a curve, $\mathcal{J}_{Y / X}$ (or $J_{Y}$ if there is no ambiguity) denotes the ideal sheaf of $Y$ in $X$ and $\omega_{Y}$ its dualizing sheaf. If $\mathcal{F}$ is a sheaf on a variety $X$ we write $h^{i}(\mathcal{F})$ for $\operatorname{dim} H^{i}(X, \mathbb{F})$. When $\operatorname{Pic}(X) \cong \mathbb{Z}$ (as in the case of smooth quartic hypersurfaces in $\mathbb{P}^{4}$ ) we use this isomorphism to identify line bundles with integers; in particular, for any vector bundle $\mathcal{E}$ we consider $c_{1}(\mathcal{E})=c_{1} \in \mathbb{Z}$ and write $\mathcal{E}(n)$ for $\mathcal{E} \otimes \mathcal{O}_{X}(n)$; also since $H^{4}(X, \mathbb{Z}) \cong \mathbb{Z}$ is generated by the class of a line, we identify $c_{2}(\mathcal{E})=c_{2} \in \mathbb{Z}$ according to this isomorphism. A locally complete intersection curve $Y \subset X$ is called $a$-subcanonical if $\omega_{Y}=\mathcal{O}_{Y}(a)$ for some $a \in \mathbb{Z}$.

We also define the number:

$$
b(\mathcal{E})=b=\max \left\{n \mid h^{0}(\mathcal{E}(-n)) \neq 0\right\} .
$$

We say that a rank-two vector bundle $\mathcal{E}$ on $X$ "splits" if it is isomorphic to the direct sum of two line bundles.

We use the notion of stability as given in [19]. When $\operatorname{Pic}(X) \cong \mathbb{Z}$, using our notation, a rank-two vector bundle $\mathcal{E}$ is stable if and only if $2 b-c_{1}<0$.

Note that the number $2 b-c_{1}$ is invariant by twisting i.e

$$
2 b-c_{1}=2 b(\mathcal{E}(n))-c_{1}(\mathcal{E}(n)) \forall n \in \mathbb{Z} .
$$


If $b=0$, as remarked in [12] (Remark 1.0.1), then $\mathcal{E}$ has a global section whose zero-locus has codimension 2 .

Definition. A rank-two vector bundle $\mathcal{E}$ on a non singular hypersurface $X$ in $\mathbb{P}^{4}$ has no intermediate cohomology if and only if $h^{1}(X, \mathcal{E}(n))=$ $0 \forall n \in \mathbb{Z}$.

We recall Riemann-Roch theorem for rank-two vector bundles on non singular hypersurfaces in $\mathbb{P}^{4}$ :

Theorem (Riemann-Roch). Let $X$ be a non-singular hypersurface in $\mathbb{P}^{4}, r$ its degree. Let $\mathcal{E}$ be a rank-two vector bundle on $X$. Then:

$$
\begin{aligned}
\chi(\mathcal{E}) & =\operatorname{deg}\left(\operatorname{ch}(\mathcal{E}) \cdot T d\left(T_{X}\right)\right)_{3}=\frac{c_{1}^{3} r}{6}+\frac{5-r}{4} c_{1}^{2} r-\frac{5-r}{2} c_{2}- \\
& -\frac{c_{1} c_{2}}{2}+\frac{r c_{1}}{12}\left((5-r)^{2}+\left(10-5 r+r^{2}\right)\right)+\frac{r}{12}(5-r)\left(10-5 r+r^{2}\right)
\end{aligned}
$$

Remark 1. Assume $r=4$, then for line bundles we have

$$
\chi\left(\mathcal{O}_{X}(t)\right)=1+\frac{7}{3} t+t^{2}+\frac{2}{3} t^{3}
$$

while for rank-two bundles

$$
\chi(\mathcal{E})=2+\frac{7}{3} c_{1}-\frac{c_{1} c_{2}}{2}-\frac{c_{2}}{2}+c_{1}^{2}+\frac{2}{3} c_{1}^{3}
$$

and by standard computation

$$
\begin{aligned}
\chi(\mathcal{E}(n)) & =2+\frac{14}{3} n+2 n^{2}+\frac{4}{3} n^{3}+\frac{7}{3} c_{1}-\frac{c_{1} c_{2}}{2}+2 n^{2} c_{1}+2 n c_{1}+2 n c_{1}^{2}+ \\
& +c_{1}^{2}+\frac{2}{3} c_{1}^{3}-n c_{2}-\frac{c_{2}}{2}
\end{aligned}
$$

\section{Examples and the proof of the main theorem}

Let $X$ be a general quartic hypersurface in $\mathbb{P}^{4}$, so that $X$ is smooth and $\operatorname{Pic}(X) \cong \mathbb{Z}$.

We start this section giving examples of non-splitting rank-two vector bundles on $X$ without intermediate cohomology. We will use the 
Hartshorne-Serre correspondence between rank-two bundles and locally complete intersection subcanonical curves on $X$ (see [10] or [24] for example). In particular we recall the exact sequence defining such a bundle

$$
0 \rightarrow \mathcal{O}_{X} \rightarrow \mathcal{E} \rightarrow \mathcal{J}_{C}\left(c_{1}\right) \rightarrow 0
$$

where $c_{1}(\mathcal{E})=c_{1}, c_{2}(\mathcal{E})=\operatorname{deg}(C)$ and $C$ is the zero-locus of a global section $s \in H^{0}(\mathcal{E})$. Note that since $h^{1}\left(\mathcal{O}_{X}(n)\right)=h^{2}\left(\mathcal{O}_{X}(n)\right)=0$ for all $n \in \mathbb{Z}$ then $h^{1}\left(\mathcal{J}_{C}\left(c_{1}+n\right)\right)=h^{1}(\mathcal{E}(n))$ for all $n \in \mathbb{Z}$. It is obvious that the rank-two bundle $\mathcal{E}$ splits if and only if the curve $C$ above is a complete intersection in $X$ of two divisors. In particular in this case $C$ must be the intersection of $X$ with two other hypersurfaces.

Example 1. By [23] or [26] there is a line contained in a general quartic hypersurface $X$. So let $l \subset X$ be a line and let $\mathcal{E}$ be the associated bundle on $X$. Then $c_{1}(\mathcal{E})=-1, c_{2}(\mathcal{E})=1, b=0$, and $\mathcal{E}$ is non-splitting (for $l$ is not complete intersection in $X$ ) without intermediate cohomology (by (1), for $l$ is arithmetically normal).

Example 2. By [26] there is a conic $C$ contained in $X$. Let $\mathcal{E}$ be the associated bundle on $X$, then $c_{1}(\mathcal{E})=0, c_{2}(\mathcal{E})=2, b=0$ and $\mathcal{E}$ is non-splitting without intermediate cohomology.

Example 3. Let $C$ be a plane cubic contained in $X$, and $\mathcal{E}$ be the corresponding bundle on $X$. Then $\mathcal{E}$ is non-splitting without intermediate cohomology with $2 b-c_{1}=-1$ and $c_{2}=3$. If $C$ is a quartic space curve, complete intersection of two quadrics, and $C$ is contained in $X$ then the bundle associated to $C$ on $X$ is non-splitting without intermediate cohomology (see Remark 4). Again for such a bundles we find $2 b-c_{1}=-1$ and $c_{2}=4$. In the same way for the bundle associated to a quintic curve of arithmetic genus 1 and trivial dualizing sheaf, we find $2 b-c_{1}=-1$ and $c_{2}=5$ (the existence of such curves on $X$ is proved in Section 3).

Example 4. Let $C$ be a smooth canonical curve (in $\mathbb{P}^{4}$ ) of degree 8 and genus 5 contained in $X$ (the existence of such curves in $X$ is proved in Section 3.). The rank-two bundle $\mathcal{E}$ associated to $C$ is non-splitting since $C$ is not complete intersection in $X$ by degree reasons, and has no intermediate cohomology. For such a bundle we find $c_{1}=2, c_{2}=8$, $b=0$.

Example 5. Let $C \subset X$ be a half-canonical curve (i.e $\omega_{C} \cong \mathcal{O}_{C}(2)$ ) of genus 15 and degree 14 (see [14] and [5]). Let $\mathcal{E}$ be the associated bundle. 
Then, by Proposition 8.2 in [5], $\mathcal{E}$ has no intermedite cohomology and, since $C$ is not contained in any quadrics, then by the sequence (1) it follows $h^{0}(\mathcal{E}(-1))=0$. For such bundles one gets $2 b-c_{1}=-3$.

Remark 2. The existence of elliptic quintic curves or the existence of smooth canonical curves on a general quartic hypersurface $X$ is proved in Section 3., Theorem 3.1 and Theorem 3.2. Also we show the existence of elliptic quartic curves on $X$ in Theorem 3.3. So the bundles constructed in Example 4 and Example 3 actually exist on such an $X$.

The existence of a half-canonical curve of degree 14 and genus 15 on a general quartic hypersurface is proved in [14]. In fact, the proof contained there is non-constructive; indeed Iliev and Markushevich prove that a general quartic hypersurface $X \subset \mathbb{P}^{4}$ is described by the Pfaffians of a suitable matrix of forms; Beauville pointed out in [5] that this fact implies the existence of the quoted curves on $X$.

Proof of the Main Theorem. The previous examples and the previous remark dispose of the existence part of the Main Theorem. So we need to prove the classification.

Let $\mathcal{E}$ be a non-splitting rank-two bundle without intermediate cohomology on $X$. By [17] for such a bundle we have $-4<2 b-c_{1}(\mathcal{E})<2$, and since the number $2 b-c_{1}$ is invariant by twist, we may assume $b=0$, hence $-4<-c_{1}<2$. We will prove theorem by a case by case analysis.

(1) If $c_{1}=-1$ since $\mathcal{E}$ has no intermediate cohomology, $\chi(\mathcal{E}(1))=$ $6-c_{2}=h^{0}(\mathcal{E}(1))=5$ hence $c_{2}=1$ and there is a global section of $\mathcal{E}$ vanishing on a line as in Example 1.

(2) If $c_{1}=0, \chi(\mathcal{E})=2-\frac{c_{2}}{2}=h^{0}(\mathcal{E})=1$ hence $c_{2}=2$. Since $h^{0}(\mathcal{E}(1))=7$ by the exact sequence (1) $C$ is contained in two independent hyperplanes so $C$ is a (possibly singular) plane conic and $\mathcal{E}$ is a bundle described in Example 2.

(3) If $c_{1}=1$ since $\chi(\mathcal{E})=6-c_{2}=h^{0}(\mathcal{E})=1+h^{0}\left(J_{C}(1)\right) \geq 1$ it is $c_{2} \leq 5$. It is clear that $c_{2}>2$, otherwise we have a global section vanishing on a line or on a plane conic. Let us consider the cases, $c_{2}=3$, $c_{2}=4$ and $c_{2}=5$. In the first one $C$ is contained in two independent hyperplanes in $\mathbb{P}^{4}$ hence $C$ is a (possibly singular) plane cubic. In the second one $C$ is a curve of degree 4 and arithmetic genus 1 , contained in one hyperplane in $\mathbb{P}^{4}$, hence, by Horrocks criterion, $C$ is the complete intersection of two quadric surfaces in $\mathbb{P}^{3}$. Finally if $c_{2}=5, C$ is a curve of degree 5 and arithmetic genus 1 in $X$. In any case we obtain one of 
the bundles constructed in Example 3.

(4) If $c_{1}=2$ then $c_{2}=8$. Indeed we find $\chi(\mathcal{E}(-1))=0$ and, being

$$
\chi(\mathcal{E}(-1))=-2+\frac{7}{3} c_{1}-\frac{c_{1} c_{2}}{2}-c_{1}^{2}+\frac{2}{3} c_{1}^{3}+\frac{c_{2}}{2}
$$

it follows $c_{2}=8$. Hence $\mathcal{E}$ is associated to a curve of degree 8 and genus 5, whose dualizing sheaf is the restriction of $\mathcal{O}_{X}(1)$; when $\mathcal{E}$ is globally generated, then we can take $C$ smooth, so that $\mathcal{E}$ is the bundle constructed in Example 4. We will go back to this situation in the next Proposition.

(5) If $c_{1}=3$ arguing as above we find $c_{2}=14$. One computes $h^{3}(\mathcal{E}(-3))=h^{0}(\mathcal{E}(-1))=0$; since the other cohomological groups vanish, by Castelnuovo-Mumford criterion the bundle $\mathcal{E}$ is globally generated hence the zero-locus $C$ of a global section $s \in H^{0}(\mathcal{E})$ is a smooth subcanonical curve of degree 14 and genus 15 and $\mathcal{E}$ a bundle described in Example 5.

Next, we still have to understand completely the case $c_{1}=2$ and $c_{2}=8$. Here we constructed in Example 4 a non-splitting rank-two vector bundle with such Chern classes, starting with a complete intersection (smooth) canonical curve; observe that we could start even with a trigonal canonical curve contained in some $X$, which is not complete intersection for it lies on a cubic scroll. The following Proposition explains what distinguishes the two types of bundles one can get.

Proposition 2.1. Let $\mathcal{E}$ be a non-splitting rank-two vector bundle without intermediate cohomology on $X$ with $c_{1}=2, c_{2}=8$ and $b=0$. Then $\mathcal{E}$ is associated to a smooth complete intersection of type $(2,2,2)$ in $\mathbb{P}^{4}$ if and only if it is globally generated.

Proof. By the previous theorem $\mathcal{E}$ is associated to a subcanonical curve of degree 8 and genus 5 , say $C$, contained in $X$. Furthermore, by Riemann-Roch $h^{0}(\mathcal{E})=4$, so by the exact sequence

$$
0 \rightarrow \mathcal{O}_{X} \rightarrow \mathcal{E} \rightarrow \mathcal{J}_{C}(2) \rightarrow 0
$$

we find $h^{0}\left({ }^{\prime} C(2)\right)=3$. Let $Q_{1}, Q_{2}, Q_{3}$ be three independent quadrics determined by such global sections and consider the intersection $Y=$ $Q_{1} \cap Q_{2} \cap Q_{3}$. 
Assume that $Y=C$. Then $J_{C}(2)$ is generated by global sections, so that also $\mathcal{E}$ is generated by global sections, for the map $H^{0}(\mathcal{E}) \rightarrow$ $H^{0}\left(\mathcal{J}_{C}(2)\right)$ surjects.

Conversely, assume that $\mathcal{E}$ is generated by global sections. Then also $J_{C}(2)$ is generated by global sections and the zero-locus of a general section of $\mathcal{E}$ is smooth (see Section 1. in [18] for example). It follows immediately that $C$ is the complete intersection of three quadrics.

Remark 3. Let $\mathcal{E}$ be as in the previous proposition and assume that $C$ is smooth but not a complete intersection; hence $\mathcal{E}$ is not globally generated. Since $h^{0}(\mathcal{E})=4$ too, $C$ is contained in three quadrics and the intersection of all such quadrics contains one additional point and $C$ is contained in a cubic scroll $W$ (see [2] Proposition III.3.1).

Moreover $\mathcal{E}$ is associated to a trigonal canonical curve in $\mathbb{P}^{4}$. Indeed let us consider the intersection of $W$ with a general quartic hypersurface $X \subset \mathbb{P}^{4}$. Since $C$ is contained in $W$ and $X$, we find $X \cap W=C+C^{\prime}$ where $C^{\prime}$ is a residual quartic to $C$. Next recall that $\operatorname{Pic}(W)$ is generated by a hyperplane section $H$ of $W$ and a line $l$ of the ruling of $W$ (see [2] for example) hence $C^{\prime}=H+l$. Observe that $C+C^{\prime}$ is equivalent to $4 H$ and $12=4 H^{2}=H \cdot 4 H$, hence $12=H \cdot(H+l+C)=H^{2}+H \cdot l+H \cdot C=$ $4+H \cdot C$, since $H^{2}=3, H \cdot l=1, l^{2}=0$, so $C \cdot H=8$. Hence by

$4=l \cdot 4 H=l \cdot\left(C+C^{\prime}\right)=l \cdot(H+l+C)=l \cdot H+l^{2}+l \cdot C=1+l \cdot C$

it is $l \cdot C=3$, and $C$ is a trigonal canonical curve (in $\left.\mathbb{P}^{4}\right)$. Note that we have assumed that there exist trigonal canonical curves on $X$, or by the previous proposition bundles $\mathcal{E}$ on $X$ not globally generated.

\section{Curves on $X$}

In this section we will give some existence results for canonical curves and elliptic quintic curves on general quartic hypersurfaces $X \subset \mathbb{P}^{4}$.

Let us denote $\mathcal{H}_{d, g}$ and $\mathcal{H}_{d, g}^{X}$ the Hilbert scheme of genus $g$ and degree $d$ curves in $\mathbb{P}^{4}$ and $X$ respectively. We recall that $\mathcal{H}_{8,5}$ has two components both of dimension 36 while the local dimension of $\mathcal{H}_{5,1}$ at a general point is 25 (see [22] for example). The main ingredient of the next theorem is the following lemma. 
Lemma 3.1. Let $C \subset \mathbb{P}^{4}$ be a general complete intersection of three quadrics $q_{1}, q_{2}, q_{3} \in H^{0}\left(\mathcal{O}_{\mathbb{P}^{4}}(2)\right)$. Then for a general choice of quadrics $q_{1}^{\prime}, q_{2}^{\prime}, q_{3}^{\prime} \in H^{0}\left(\mathcal{O}_{\mathbb{P}^{4}}(2)\right)$ the quartic given by $f=\sum q_{i} q_{i}^{\prime}$ is smooth and contains $C$. Moreover the map

$$
\psi: H^{0}\left(\mathcal{O}_{\mathbb{P}^{4}}(2)\right)^{3} \rightarrow H^{0}\left(\mathcal{O}_{C}(4)\right)
$$

defined by sending $\left(g_{1}, g_{2}, g_{3}\right) \rightarrow \sum_{i} g_{i} q_{i}^{\prime}+I$ where $I=\sum_{i} q_{i} S$ and $S$ is the polinomial ring of $\mathbb{P}^{4}$, is surjective.

Proof. Globalizing and using Nakayama lemma it is sufficient to find two triples of relative prime quadrics $q=\left(q_{1}, q_{2}, q_{3}\right)$ and $q^{\prime}=\left(q_{4}, q_{5}, q_{6}\right)$ such that the map

$$
\psi: S_{2}^{3} \rightarrow(S / I)_{4}
$$

defined by $\psi\left(g_{1}, g_{2}, g_{3}\right)=q_{4} g_{1}+q_{5} g_{2}+q_{6} g_{3}+I$, where $I=\sum_{i=1}^{3} q_{i} S$, is surjective. Let $q_{1}=x_{1}^{2}, q_{2}=x_{2}^{2}, q_{3}=x_{3}^{2}$ and $q_{4}=x_{4}^{2}, q_{5}=x_{1} x_{2}-x_{5}^{2}$, $q_{6}=x_{3} x_{4}-x_{5}^{2}$. Note that $(S / I)_{4}$ is spanned by monomials of the form

$$
f_{4}, x_{1} f_{3}, x_{2} f_{3}, x_{3} f_{3}, x_{1} x_{2} f_{2}, x_{1} x_{3} f_{2}, x_{2} x_{3} f_{2}, x_{1} x_{2} x_{3} f_{1}
$$

where the $f_{i}$ 's are monomials in $x_{4}, x_{5}$ of degree $i$. Now, just making the computations, one finds the previous map is surjective.

In fact, these computations were originally performed by means of the computer algebra package $\mathrm{CoCoA}[7]$.

We are now able to show

Theorem 3.1. Let $X \subset \mathbb{P}^{4}$ be a general quartic hypersurface. Then:

1. there exists a smooth curve $C \subset X$ of genus 5 and degree 8;

2. $h^{1}\left(\mathcal{N}_{C / X}\right)=0$ and hence $h^{0}\left(\mathcal{N}_{C / X}\right)=8$ and $\mathcal{H}_{8,5}^{X}$ is smooth of local dimension 8 at $[C]$.

Proof. Let $I=\left\{(C, X): C \subset X, C \in \mathcal{H}_{8,5}, X \in\left|\mathcal{O}_{\mathbb{P}^{4}}(4)\right|\right\}$ and consider 
the projections

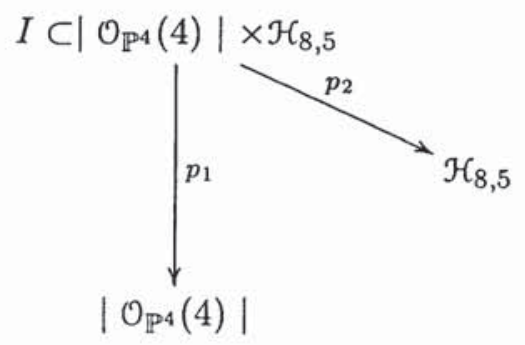

Let $C$ be a general point of the component parametrizing canonical curves, then $C$ is a smooth complete intersection of type $(2,2,2)$ (see [2]). Since $h^{0} \mathcal{J}_{C / \mathbb{P}^{4}}(4)=42$ it follows $\operatorname{dim}(I)=77$. If $h^{1}\left(\mathcal{N}_{C / X}\right)=0$, since $\chi\left(\mathcal{N}_{C / X}\right)=h^{0}\left(\mathcal{N}_{C / X}\right)=8$, the projection $p_{1}$ is dominant. Hence we have to find a smooth canonical curve $C_{o}$ and a smooth quartic hypersurface $X=X_{o}$ containing $C=C_{o}$ such that $h^{1}\left(\mathcal{N}_{C / X}\right)=0$. For a general choice of quadrics $q_{1}, q_{2}, q_{3}$ the curve complete intersection of such quadrics is smooth and for general quadrics $q_{1}^{\prime}, q_{2}^{\prime}, q_{3}^{\prime}$ the quartic hypersurface given by $f=\sum_{i} q_{i} q_{i}^{\prime}$ is smooth and contains $C$. Since $C$ is a complete intersection in $\mathbb{P}^{4}$ we find $\mathcal{N}_{C / \mathbb{P}^{4}}=\mathcal{O}_{C}(2)^{\oplus 3}$. Consider the standard exact sequence

$$
0 \rightarrow \mathcal{N}_{C / X} \rightarrow \mathcal{O}_{C}(2)^{\oplus 3} \rightarrow \mathcal{O}_{C}(4) \rightarrow 0 .
$$

By the previous lemma we may assume the surjectivity of the map

$$
H^{0}\left(\mathcal{O}_{\mathbb{P}^{4}}(2)\right)^{\oplus 3} \rightarrow H^{0}\left(\mathcal{O}_{C}(4)\right) .
$$

Since such map factors through the map $\psi: H^{0}\left(\mathcal{O}_{C}(2)\right)^{\oplus 3} \rightarrow H^{0}\left(\mathcal{O}_{C}(4)\right)$, this last is surjective too; then $h^{1}\left(\mathcal{O}_{C}(2)\right)=0$ implies $h^{1}\left(\mathcal{N}_{C / X}\right)=0$.

Next we will consider the case of elliptic curves on $X$.

Theorem 3.2. Let $X \subset \mathbb{P}^{4}$ be a general quartic hypersurface. Then:

1. there exists a quintic curve $C \subset X$ of genus 1 and trivial dualizing sheaf;

2. $h^{1}\left(\mathcal{N}_{C / X}\right)=0$ and hence $h^{0}\left(\mathcal{N}_{C / X}\right)=5$ and $\mathcal{H}_{5,1}^{X}$ is smooth of local dimension 5 at $[C]$. 
Proof. Consider the Hilbert scheme $\mathcal{H}_{5,1}$ of elliptic quintics in $\mathbb{P}^{4}$. The dimension of such a scheme at a general point is 25 , (see [22] for example). Next consider the incidence scheme $I=\{(C, X): C \subset X, X \in$ $\left.\left|\mathcal{O}_{\mathbb{P}^{4}}(4)\right|,[C] \in \mathcal{H}_{5,1}\right\}$ and the following diagram:

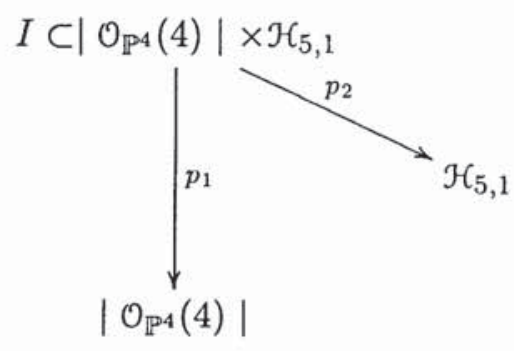

The dimension of the fibre of the map $p_{2}$ is $h^{0} \mathcal{J}_{C}(4)$, so by RiemannRoch it is 49-dimensional; it follows that the dimension of the incidence scheme is 74 . To prove the existence of an elliptic quintic on $X$ it is sufficient to show that the fibre of the projection $p_{1}$ at a general point $[X]$ is 5 -dimensional, indeed in such a case the map is dominant. If we show that $h^{1}\left(\mathcal{N}_{C / X}\right)=0$ then we are done since in this case the dimension of such a fibre is given by $h^{0}\left(\mathcal{N}_{C / X}\right)=\chi\left(\mathcal{N}_{C / X}\right)=5$. To show the previous vanishing, by the exact sequence

$$
0 \rightarrow T_{C} \rightarrow T_{X \mid C} \rightarrow \mathcal{N}_{C / X} \rightarrow 0
$$

it is sufficient to show that $h^{1}\left(T_{X \mid C}\right)=0$. Look at the exact sequence

$$
0 \rightarrow T_{X \mid C} \rightarrow T_{\mathbb{P}^{4} \mid C} \rightarrow \mathcal{O}_{C}(4) \rightarrow 0
$$

and consider the maps $\beta: \mathcal{O}_{C}(1)^{\oplus 5} \rightarrow T_{\mathbb{P}^{4} \mid C}$ and $\alpha: \mathcal{O}_{C}(1)^{\oplus 5} \rightarrow \mathcal{O}_{C}(4)$, where $\beta$ is the restriction of the Euler map, and the map $\alpha$ is defined by the 5 derivatives of an equation of $X$ (the so-called Jacobian map of $X$, restricted to $C$ ).

We are done if we prove that $\alpha$ surjects in global sections, for in this case also $H^{0}\left(T_{\mathbb{P}^{4} \mid C}\right) \rightarrow H^{0}\left(\mathcal{O}_{C}(4)\right)$ is surjective, while on the other hand $H^{1}\left(T_{\mathbb{P}^{4} \mid C}\right)=0$ by the Euler sequence, for $H^{1}\left(\mathcal{O}_{C}(1)\right)=0$. To prove the surjectivity, we just compute the intersection of the homogeneous ideal of $C$ with the Jacobian ideal of the derivatives of $X$; one needs the intersection to be 65 dimensional, for in this case, in global sections, $\alpha$ has rank $h^{0}\left(\mathcal{O}_{\mathbb{P}^{4}}(4)\right)-65=5=h^{0}\left(\mathcal{O}_{C}(4)\right)$. 
The surjectivity of $\alpha$ was checked directly: by the computer package [20] we got (quadric) generators for the homogeneous ideal of a general elliptic quintic curve $C$. Then we took a random quartic form $X$ through $C$ and made derivatives; finally by means of the computer package [7] we obtained the dimension of the intersection of the Jacobian ideal of $X$ with the ideal of $C$, in degree 4 (see Remark 5).

Theorem 3.3. Let $X \subset \mathbb{P}^{4}$ be a general quartic hypersurface. Then:

1. there exists a quartic curve $C \subset X$ of genus 1 and trivial dualizing sheaf;

2. $h^{1}\left(\mathcal{N}_{C / X}\right)=0$ and hence $h^{0}\left(\mathcal{N}_{C / X}\right)=4$ and $\mathcal{H}_{4,1}^{X}$ is smooth of local dimension 4 at $[C]$.

Proof. Elliptic quartic curves $\mathrm{C}$ are complete intersections of type $(1,2,2)$ in $\mathbb{P}^{4}$. So, one can use the same procedure as in theorem 3.1 to get the result. Alternatively one can use once again the procedure of theorem 3.2 (which in this case simplifies for the equations for $\mathrm{C}$ are just one general hyperplane and two general quadrics) to prove that a general quartic hypersurface contains a 4-dimensional family of elliptic quartic curves.

Remark 4. In the previous theorem we proved that a general quartic hypersurface $X$ contains a 4-dimensional family of elliptic quartic curves. Notice however that a general hyperplane section $Y$ of $X$ does not contain curves like $C$ since $\operatorname{Pic}(Y)=\mathbb{Z}[h], h$ being the hyperplane class of $Y$, so it represents a quartic of arithmetic genus 3 . Instead, there exists a 3-dimensional family of hyperplane sections of $X$ containing a smooth quartic elliptic curve. Observe in fact that, by a standard Riemann-Roch computation, if a hyperplane section contains an elliptic quartic, then it contains a pencil of such curves. All these pencils together describe the required 4-dimensional family of elliptic quartics.

Remark 5. For the sake of completeness, we add here the homogeneous ideal of the elliptic curve $C$ we used in the previous computations perfomed in $\mathbb{Z} /(32003)$ 
$\left(x_{1} x_{2}-13465 x_{2}^{2}+2446 x_{0} x_{3}+11548 x_{1} x_{3}-10808 x_{2} x_{3}-14478 x_{3}^{2}+\right.$ $12936 x_{0} x_{4}-9813 x_{1} x_{4}+11887 x_{2} x_{4}+7482 x_{3} x_{4}-9596 x_{4}^{2}, x_{0} x_{2}+5409 x_{2}^{2}+$ $5355 x_{0} x_{3}+6803 x_{1} x_{3}+14831 x_{2} x_{3}-14803 x_{3}^{2}-2249 x_{0} x_{4}+7415 x_{1} x_{4}+$ $1199 x_{2} x_{4}+3610 x_{3} x_{4}-15434 x_{4}^{2}, x_{1}^{2}-14637 x_{2}^{2}-4510 x_{0} x_{3}+3148 x_{1} x_{3}+$ $7884 x_{2} x_{3}-624 x_{3}^{2}-8857 x_{0} x_{4}-11501 x_{1} x_{4}-8624 x_{2} x_{4}-3708 x_{3} x_{4}+$ $10856 x_{4}^{2}, x_{0} x_{1}-1484 x_{2}^{2}-15834 x_{0} x_{3}-2034 x_{1} x_{3}-3036 x_{2} x_{3}-140 x_{3}^{2}+$ $6790 x_{0} x_{4}+6935 x_{1} x_{4}+12529 x_{2} x_{4}-9374 x_{3} x_{4}+4473 x_{4}^{2}, x_{0}^{2}-6169 x_{2}^{2}+$ $3429 x_{0} x_{3}-10569 x_{1} x_{3}-12912 x_{2} x_{3}-6022 x_{3}^{2}-735 x_{0} x_{4}+10572 x_{1} x_{4}-$ $\left.9979 x_{2} x_{4}-8807 x_{3} x_{4}+6714 x_{4}^{2}\right)$

All the computations performed by means of computer algebra packages were in fact tested only in some characteristic $p>0$; however, since in all cases we needed just to prove that a natural map has maximal rank, a positive answer in any characteristic implies a positive answer in characteristic 0 .

\section{References}

[1] R. Apéry Sur certain caractéres numeriques d'un idéal sans composant impropre, C. R. Acad. Sci. Paris 220 (1945), 234-236. Sur les courbes de prémiere espéce de l'espace à trois dimensions, C. R. Acad. Sci. Paris 220 (1945), 271-272.

[2] E. Arbarello, M. Cornalba, P.A. Griffiths and J. Harris, Geometry of algebraic curves I, Springer (1985).

[3] E. Arrondo and B. Graña, Vector bundles on $G(1,4)$ without intermediate cohomology, J. of Algebra 214 (1999), 128-142.

[4] E. Arrondo and L. Costa, Vector bundles on Fano 3-folds without intermediate cohomology, Comm. Algebra 28 (2000), no. 8, 3899-3911.

[5] A. Beauville, Determinantal hypersurfaces, math. AG/9910030, preprint.

[6] R.O. Buchweitz, G.M. Greuel and F.O. Schreyer, Cohen-Macaulay modules on hypersurface singularities II, Invient. Math. 88(1987), 165182.

[7] CoCoA Commutative Computer Algebra, http://cocoa.dima.unige.it.

[8] L. Costa, Vector bundles on ruled Fano 3-folds, Manuscripta Math. 100 (1999), no. 3, 335-349. 
[9] F. Gaeta, Quelques progrés récents dans la classification des variétés d'un espace prójectif, Deuxième Collogue de Géométrie Algébrique Liége, C.B.R.M. (1952).

[10] P. Griffiths and J. Harris, Two proofs of a theorem concerning algebraic space curve, Proceeding of the Eigth National Mathematics Conference (Arya-Mehr Univ. Tech., Teheran, 1977), 350-370.

[11] R. Hartshorne, Algebraic Geometry (GTM 52), Springer-Verlag Berlin, 1993.

[12] Stable vector bundles of rank 2 on $\mathbb{P}^{3}$, Math. Ann. 238(1978), 229-280.

[13] G. Horrocks, Costruction of bundles on $\mathbb{P}^{n}$, in: Séminaire DouadyVerdier E.N.S. 77/78, Asterisque 71-72, 197-203.

[14] A. Iliev and D. Markushevich, Quartic 3-fold: Pfaffians, instantons and half-canonical curves, preprint math.AG/9910133.

[15] H.P. Kley, Rigid curves in complete intersection Calabi-Yau threefolds, Compositio Math., to appear.

[16] H. Knörrer, Cohen-Macaulay modules on hypersurface singularities I, Inv. Math. 88(1987), 153-164.

[17] C. Madonna, A splitting criterion for rank 2 vector bundles on hypersurfaces in $\mathbb{P}^{4}$, Rend. Sem. Mat. Univ. Politec. Torino, to appear.

[18] S. Mukai, Polarized K3 surfaces of genus 18 and 20 in: Complex projective geometry, 264-276, London Math.Soc.Lecture Note Ser., 179, Cambridge Univ.Press, Cambridge, 1992.

[19] C. Okonek, M. Schneider and H. Spindler, Vector Bundles on Complex Projective Spaces (Prog. Math. 3), Birkhäuser, Boston, Mass.,1980.

[20] F. Orecchia, L. Chiantini, F. Cioffi and I. Ramella, Elliptic computer package (in preparation).

[21] G. Ottaviani, Some extension of Horrocks criterion to vector bundles on Grassmannians and quadrics, Ann. Mat. Pura Appl. (4) 155 (1989), 317-341.

[22] E. Sernesi, Topics on Families of Projective Schemes, Queens Papers Math. (1986).

[23] V.V. Sokurov, The existence of a straight line on Fano 3-folds, Math.USSR Izvestija Vol. 15 (1979) no.1, 173-209 (1980).

[24] L. Szpiro, Lectures on equations defining space curves, Tata Institute of Foundamental Research Bombay, Springer, 1979. 
[25] M. Szurek and J.A. Wiśniewski, Conics, conic fibrations and stable vector bundles of rank 2 on some Fano threefolds, Rev. Roumaine Math. Pures Appl., 38 (1993), 7-8, 729-741.

[26] B.R. Tennison, On the quartic threefold, Proc.London Math.Soc. (3) 29 (1974), 714-734.

Dipartimento di Matematica

Università di Roma Tor Vergata

Viale della Ricerca Scientifica

00133 Roma

Italy

E-mail: madonna@mat.uniroma2.it

Recibido: 29 de Noviembre de 1999

Revisado: 29 de Mayo de 2000 